A N N A L E S Annales de Bretagne et des Pays de l'Ouest

Anjou. Maine. Poitou-Charente. Touraine

$122-4 \mid 2015$

Varia

\title{
Villes de Bretagne
}

\section{Olivier Charles}

\section{OpenEdition}

Journals

Édition électronique

URL : https://journals.openedition.org/abpo/3180

DOI : $10.4000 / a b p o .3180$

ISBN : 978-2-7535-4882-4

ISSN : 2108-6443

Éditeur

Presses universitaires de Rennes

Édition imprimée

Date de publication : 15 décembre 2015

Pagination : 163-166

ISBN : 978-2-7535-4880-0

ISSN : 0399-0826

Référence électronique

Olivier Charles, «Villes de Bretagne », Annales de Bretagne et des Pays de l'Ouest [En ligne], 122-4| 2015,

mis en ligne le 15 décembre 2015, consulté le 21 septembre 2021. URL : http://

journals.openedition.org/abpo/3180; DOI : https://doi.org/10.4000/abpo.3180 
nombre de militants catholiques du rouge au vert, tant à partir du Mouvement Rural de la Jeunesse Chrétienne (MRJC) que de Vie Nouvelle.

L'absence de l'horizon catholique, et surtout des racines catholiques, interroge : distraction ou dénégation? Tudi Kernalegenn raisonne sur l'écologie comme JeanJacques Monnier sur le mouvement breton : entre 1914 et 1945, il n'y a rien - ou que des choses qui fâchent. Ou presque, car il y a Émile Masson, présenté comme le " premier écologiste breton ". Masson, je veux bien, mais enfin c'est l'ancêtre à tout faire d'une gauche morale qui cherche à meubler une période plus portée vers la droite ou l'extrême droite, y compris chez les défenseurs de la nature. On se souvient qu'à Vichy la terre ne mentait pas. Et alors? Faut-il effacer ce passé? Où est le problème? On devine qu'il y en a un quand on lit, au détour d'une page, que " l'agriculture biologique s'éloigne progressivement, à partir de 1968, des fondements réactionnaires qui avaient présidé à sa naissance ". Réactionnaire, voilà, j'imagine, le péché originel de l'écologie. Question : l'immaculée conception de l'écologie est-elle un dogme?

Yvon TRANVOUEZ

Andrieux, Jean-Yves (dir.), Villes de Bretagne. Patrimoine et histoire, Rennes, PUR, 2014, 383 p.

Ce bel ouvrage collectif publié sous la houlette de Jean-Yves Andrieux, professeur d'histoire de l'art à l'université Paris-Sorbonne, propose une véritable histoire de la Bretagne urbaine. Embrassant de larges préoccupations scientifiques, patrimoniales et touristiques, il vient combler une lacune bibliographique et marquer les 40 ans de l'Association Petites Cités de Caractère de Bretagne, les 30 ans de l'Union bretonne des Villes d'art et d'histoire - d'ailleurs coéditeurs de l'ouvrage avec les Presses universitaires de Rennes -, les 25 ans de la Conférence nationale du tourisme urbain. Il rappelle aussi qu'en ces temps d'urbanisation accélérée où les politiques d'urbanisme et du patrimoine deviennent des enjeux centraux, le recours à l'histoire est plus que jamais essentiel.

L'ouvrage reflète des choix fermes de la part de ses concepteurs. Tout d'abord, il considère tout l'espace de la Bretagne historique et tous les types de villes au travers d'exemples choisis de Brest à Fougères, de Lannion ou Corseul à Quiberon ou Nantes. Ensuite, il envisage son sujet sur le long terme, depuis la lente émergence du fait urbain pendant l'Antiquité jusqu'aux aménagements les plus récents, comme le " projet urbain " rennais contemporain (p. 300 et 302). Par ailleurs, cette histoire, comme l'indique le titre de l'ouvrage, est étroitement associée à une réflexion sur le patrimoine. De nombreuses contributions s'efforcent en effet de mesurer le poids du bâti ancien - les murailles n'en étant que l'illustration la plus commode -, des activités économiques - de la production de toiles à la construction navale par exemple - ou de la création ex nihilo d'un espace urbanisé - que l'on pense aux stations balnéaires -, dans le devenir des villes. Le dernier chapitre, dû à JeanBernard Vighetti, décrypte d'ailleurs le processus qui a conduit, tardivement mais résolument, nombre de villes à associer préservation du patrimoine et promotion du tourisme, une des sources de richesse de la région, sous l'impulsion des réseaux cités ci-dessus. Le défi, relevé, était de taille : éviter que les cités concernées ne deviennent des villes-musées en promouvant " une politique identifiée de sauvegarde, d'entretien, de valorisation et d'animation du patrimoine " (p. 341). Enfin, Villes de Bretagne ménage une place de choix à l'iconographie (inscriptions, recons- 
titutions, plans, cartes postales, photographies, affiches, tableaux...). Il s'agit d'une orientation raisonnée, jamais gratuite, dans la mesure où les légendes ne sont pas redondantes mais apportent au texte lui-même. On mesure ainsi l'ambition et l'importance de cette première synthèse sur les villes bretonnes.

En seize thèmes classés en quatre grandes périodes plus une réflexion portant sur " La ville symbolique", le lecteur parcourt toute l'histoire urbaine de la Bretagne. Au fil de la plume de spécialistes, historiens pour beaucoup, mais aussi historiens d'art ou aménageurs, il est ainsi confronté tout au long d'une étude chrono-thématique de longue durée aux acquis récents de la recherche. Les réflexions sur la paroisse urbaine aux temps modernes, sur le façonnement de la ville par les parlementaires (Rennes, Vannes), sur la ville contemporaine ne sont en la matière que quelques exemples laissant supposer la richesse de l'ouvrage. Une première partie étudie la " Naissance de la ville ". Passée la période antique qui voit se dessiner un premier réseau urbain, l'époque médiévale est celle du façonnement de la ville par l'Église, les seigneurs, les ducs, dans le cadre d'une évolution qui est loin d'être linéaire. Yvan Maligorne insiste sur le regard archéologique neuf que permettent les nombreuses fouilles engagées depuis une vingtaine d'années et le recours croissant aux comparaisons. Notre connaissance des origines des villes s'en trouve à la fois renouvelée et approfondie. Yves Coativy rappelle quant à lui qu'après les troubles qui accompagnent et suivent la fin de l'Empire romain une soixantaine de villes s'affirment progressivement à partir du XI ${ }^{\mathrm{e}}$ siècle, bien souvent au pied d'un château, mais que la guerre de Succession remet en cause leur croissance. Dans une perspective à la fois historique et patrimoniale, Daniel Pichot étudie ensuite les relations que les villes entretiennent avec leurs murailles entre le XIV ${ }^{\mathrm{e}}$ et le XXI ${ }^{\mathrm{e}}$ siècle. Tout en prolongeant les réflexions du chapitre précédent, il montre comment elles ont d'abord été une nécessité (la protection) avant d'être porteuses d'identité (le pouvoir) puis de mémoire. En cela, « le paysage de la ville n'est que la recomposition des legs divers, une actualisation de l'histoire " (p. 65).

Dans la seconde partie de l'ouvrage, nous passons en quatre chapitres " De la ville médiévale à la ville classique ". L'étude de la ville religieuse est l'occasion pour Georges Provost de rappeler au lecteur que le renouvellement tridentin fait du clocher un symbole au moins aussi important que les murailles, symbole que les villes s'efforceront plus tard de valoriser pour des raisons identitaires et patrimoniales. Cette époque est par ailleurs celle du renforcement de l'emprise monumentale, spatiale et sociale de l'Église sur l'espace urbain. C'est aussi celle de l'épanouissement d'un certain nombre de cités grâce à l'essor de l'activité textile comme le montrent André Lespagnol et Jean Martin en évoquant la ville toilière et négociante. Canevas, olonnes, crées et autres bretagnes sont en effet à l'origine d'importants patrimoines architecturaux non seulement dans les grandes villes portuaires que sont Morlaix ou Saint-Malo mais aussi dans une quantité de petites villes de l'intérieur. Gauthier Aubert présente ensuite la ville parlementaire (Rennes certes, mais aussi, brièvement il est vrai, Vannes), ce qui lui permet de proposer une histoire socio-spatiale de Rennes devenue " vraiment la capitale de la Bretagne, par la concentration des centres de décision politique, militaire et judiciaire " (p. 114) à la charnière des XVII ${ }^{\mathrm{e}}$ et XVIII ${ }^{\mathrm{e}}$ siècles. Dominée par l'imposant palais du parlement aménagé, transformé, restauré jusqu'à aujourd'hui, façonnée pour longtemps par les demeures de parlementaires formant cependant une élite en partie détachée de la ville, Rennes s'affirme comme une ville à la fois française et bretonne. Enfin, Guy Saupin s'attache à l'étude de la ville modèle, rappelant que l'époque des Lumières, fortement influencée par une esthétique formulée dès la Renaissance, se fixe un programme ambitieux : l'embellissement, le remodelage, l'aération de l'espace urbain. C'est l'époque de l'affirmation de la ville centre de 
commandement au détriment de la ville statutaire. Le principe de la circulation sans entrave des hommes - marchands comme militaires -, des biens et de l'air, appliqué dans bien des cités, petites ou grandes, triomphe avec la construction de quartiers neufs (à Rennes après l'incendie de 1720 par exemple) ou l'édification de villes neuves (Brest ou Lorient).

L'examen de "La ville du XIX ${ }^{e}$ siècle " permet ensuite de rappeler les lignes de force de la période au détour de quatre chapitres. Pascal Burguin présente les transformations spectaculaires de la physionomie urbaine à l'époque du libéralisme économique dominant. Alors que se renouvèlent leurs fonctions traditionnelles encadrer, redistribuer, loger -, les villes connaissent de profondes mutations sous l'impulsion des élites bourgeoises : étalement, embellissement, rationalisation, équipement. Claude Geslin rappelle quant à lui que c'est véritablement au XIX siècle que l'industrie investit réellement, quoique inégalement, les villes bretonnes, suscitant l'apparition d'une nouvelle population et de nouveaux paysages. Déployant l'analyse en aval, il rappelle que si les années 1960-1970 marquent la disparition des quartiers populaires, les villes s'interrogent aujourd'hui, après les espoirs nés de la décentralisation, sur l'avenir de leurs activités industrielles. La présentation de la ville portuaire permet à Gérard Le Bouëdec d'étudier ensuite le long processus qui a conduit la kyrielle de petits ports de pêche et de cabotage du littoral breton à se muer en ports de plaisance pendant que les grands ports marchands et militaires des XVII et XVIII ${ }^{\mathrm{e}}$ siècles - Nantes, Lorient, Brest - s'adaptaient à la nouvelle donne. Le littoral est aussi modifié par la naissance de la ville balnéaire qu'examine JeanYves Andrieux. L'anglomanie dont font preuve les élites ainsi que l'essor du chemin de fer - puis de l'automobile - et la nouvelle image, positive, dont jouit le littoral conduisent à l'apparition de ces villes de villégiature à l'urbanisme et à l'architecture si particuliers. Quant à " La ville du Xx $x^{e}$ siècle ", quatrième partie de l'ouvrage, elle est étudiée en trois séquences chronologiques. Tout d'abord, Philippe Bonnet explore les voies de la modernité et les premiers temps de la planification urbaine jusqu'en 1940. Ensuite, Patrick Dieudonné étudie les modèles de la reconstruction au lendemain de la Seconde Guerre mondiale, avant que Daniel Le Couédic ne réfléchisse au paradoxe du $\mathrm{xx}^{\mathrm{e}}$ siècle urbaniste, critiqué pour son " insouciance de l'héritage recueilli " (p. 282) mais crédité d'un réel souci de protection et de mise en valeur du patrimoine.

On pourrait regretter à ce stade l'absence de synthèses thématiques ou de période. Mais le choix initial et original de focaliser la réflexion sur un ou plusieurs traits dominants par époque, sur des types de ville, tout en étirant parfois la réflexion jusqu'à aujourd'hui rend certainement cette approche difficile. Peutêtre de vigoureuses conclusions de parties auraient-elles pu néanmoins enrichir le propos, tout en conduisant à éviter certaines redites. Les chapitres consacrés à la ville fortifiée, aux villes portuaires ou à la ville industrielle en pallient d'ailleurs ponctuellement l'absence et l'avant-dernier en montre, semble-t-il, tout l'intérêt. En effet, l'étude des villes d'images et de papier est bien une proposition de lecture sur la longue durée. Patrick Harismendy démontre en effet que depuis fort longtemps la ville est aussi une réalité construite, formée d'images multiples, mouvantes, tantôt superposées, tantôt successives... Du XVII ${ }^{\mathrm{e}}$ au XXI ${ }^{\mathrm{e}}$ siècle, les récits de voyage, les romans (sans omettre les policiers), la peinture, la bande dessinée, la philatélie, le cinéma... ont en effet à la fois traduit et modelé notre imaginaire urbain.

Tout au long de l'ouvrage, le lecteur est ainsi guidé dans l'exploration de la genèse d'un système spatial particulier, aujourd'hui polarisé par trois métropoles, ponctué d'un semi de petites villes et maillé d'une multitude de bourgs ancestraux (p. 304). Villes de Bretagne confirme également, si besoin était, que la Bretagne, à 
la mesure de la longue durée, est loin d'être rétive à la modernité et, qu'au-delà des stéréotypes, elle n'est pas qu'un pays de paysans et de marins. Enfin, les deux index (noms et lieux), la table des illustrations - qui constitue en elle-même une clé de lecture de l'ouvrage - et la bibliographie font de cet ouvrage non seulement un beau livre mais aussi une synthèse de premier plan aux multiples entrées susceptibles d'intéresser un large lectorat.

Olivier CHARLES 